\title{
Capacidade funcional de usuários do pilates
}

\author{
Mikaele Romão de Oliveira ${ }^{a}$ (D) Roque Ribeiro da Silva Júnior ${ }^{b}$ (D), Daniela Maria Maia Silva ${ }^{a}$, \\ Kariza Barreto Lopes ${ }^{a}$ iD
}

${ }^{a}$ Faculdade do Vale do Jaguaribe (FVJ), Rodovia CE-040, S/№, Aeroporto, CEP: 62.800-000, Aracati, CE, Brasil. bUniversidade do Estado do Rio Grando do Norte (UERN), Rua Atirador Miguel Antonio da Silva, S/№, Aeroporto, CEP: 59.607360, Mossoro, RN, Brasil.

RESUMO O Método Pilates foi criado pelo alemão Joseph Hubertus Pilates, por volta dos anos 20, mas teve um reconhecimento por volta dos anos 40 , na cidade de Nova lorque, com um público mais assíduo de dançarinos da região. Sendo assim nos anos 80 teve um grande reconhecimento de suas técnicas, já por sua vez, nos anos posteriores seu método ficou mundialmente conhecido e difundido de forma ativa. Para uma boa execução, o Pilates adota de seis princípios a serem seguidos que são: concentração, fluidez, controle, precisão e respiração. Objetivo do estudo foi verificar a capacidade funcional e o nível de atividade física dos usuários que praticam o método Pilates. O estudo é uma pesquisa transversal de campo do tipo exploratória com abordagem quantitativa. O instrumento de coleta dos dados foi através de dois questionários, um dos foi o HAQ que mediu a capacidade funcional, contendo 20 perguntas, sendo que as alternativas para serem marcadas eram sem qualquer, com alguma, com muita e incapaz de fazer, relacionado ao grau de dificuldade para realizar a atividade. O outro questionário aplicado foi O IPAQ relacionado a atividade física, com perguntas relacionadas ao tempo gasto com relação a realização dessa atividade, sendo de leve a vigorosa. Foi possível verificar que o método pilates é bastante eficaz, contribuindo na melhora da qualidade de vida de seus praticantes assíduos, como por exemplo, melhorando a flexibilidade, força muscular, equilíbrio, alivia as dores, melhora a postura. Embora ainda se faça necessário mais estudos acerca do assunto.

PALAVRAS-CHAVE avaliação; capacidade funcional; pilates

Aceito 12 de abril de 2021 Publicado online 24 de abril de 2021

Cite este artigo: Oliveira et al. (2021) Capacidade funcional de usuários do pilates. Multidisciplinary Reviews 4: e2021012. DOI: 10.29327/multi.2021012

\section{Functional capacity of pilates users}

ABSTRACT The Pilates Method was created by the German Joseph Hubertus Pilates around the 1920s but had a recognition around the 1940s, in New York City, with a more frequent audience of dancers in the region. Thus, in the 1980s, he had a great recognition of his techniques, and in his later years, his method became known worldwide and actively disseminated. For good performance, Pilates adopts six principles to be followed, which are: concentration, fluidity, control, precision and breathing. The study aimed to verify the functional capacity and physical activity of users who practice the Pilates method. The study is cross-sectional exploratory field research with a quantitative approach. The instrument for data collection was through two questionnaires, one of which was the HAQ that measured functional capacity, containing 20 questions, and the alternatives to be marked were without any, with any, with a lot and unable to ask, related to the degree of difficulty to carry out the activity. The other questionnaire applied was the IPAQ related to physical activity, with questions about the time spent in relation to this activity, from mild to vigorous. It was possible to verify that the pilates method is quite effective, contributing to improving its regular practitioners' quality of life, such as improving flexibility, muscle strength, balance, relieving pain, and improving posture. However, further studies on the subject are still needed.

KEYWORDS: assessment; functional capacity; pilates 


\section{Introdução}

O Método Pilates foi criado pelo alemão Joseph Hubertus Pilates, por volta dos anos 20, mas teve um reconhecimento por volta dos anos 40, na cidade de Nova lorque, com um público mais assíduo de dançarinos da região. Sendo assim nos anos 80 teve um grande reconhecimento de suas técnicas, já por sua vez, nos anos posteriores seu método ficou mundialmente conhecido e difundido de forma ativa (Silva e Mannrich 2009).

O pilates é uma atividade física considerada de baixo impacto e com a sua intensidade de leve a alta, dependendo da meta que será proposta pelo método e pelas particularidades de cada indivíduo. Existe uma extensa quantidade de exercícios e variações que tornam o pilates um programa completo de condicionamento corporal e mental perfeito para diferentes pessoas (Pacheco et al 2017).

Para uma boa execução, o Pilates adota de seis princípios a serem seguidos que são: concentração, fluidez, controle, precisão e respiração (Buenos e Neves 2019). Lembrando sempre de respeitar os limites do paciente e à medida que ele vai ganhando habilidades dificulta-se o exercício (Silva e Mannrich 2009).

Os exercícios em sua grande maioria são executados na posição deitada para diminuir os impactos nas articulações que sustentam o nosso corpo quando estamos em pé. São realizadas contrações isotônicas (concêntricas e excêntricas) e isométricas baseando-se no centro de força, o mesmo é composto por quatro músculos que são: os abdominais, transverso abdominal, multífido e músculos do assoalho pélvico. Para que ocorra uma boa execução da técnica é essencial que a expiração esteja associada a contração desses músculos (Marés et al 2012).

O Pilates se divide em dois tipos de exercícios, no solo e em aparelhos, ambos fornecem um bom trabalho nos músculos estabilizadores enquanto vão eliminando a grande tensão dos músculos e compensações. Os exercícios que são executados no solo são feitos para ganhar consciência corporal, ou seja, trabalha-se a respiração e o centro de força, já nos aparelhos tem-se uma infinidade de movimentos regulando sua intensidade através de molas, porém trabalhados de maneira rítmica, controlada, sempre associando à respiração e correção postural (Pires e Sá 2005).

Independentemente da idade, qualquer indivíduo pode ter algum efeito com a prática do pilates que melhora o bem-estar. Este método corrige a flexibilidade, os desequilíbrios musculares, a postura, o condicionamento físico e a consciência corporal. Com a prática diária e da maneira correta, o pilates poderá levar a alcançar muitos benefícios tanto emocionais quanto físicos e logo melhorar a qualidade de vida de quem o pratica (Elizabete et al 2013).

A capacidade funcional é denominada como a habilidade para executar exercícios que dão a possibilidade para o indivíduo cuidar de si e viver de forma autônoma. Sua avaliação tornou-se necessária para escolher um tratamento mais eficaz. Ela pode ser verificada em dois parâmetros: com relação as ABVD (atividades básicas de vida diária) e as AIVD (atividades instrumentais de vida diária) (Pinto et al 2015).

Dentro do que foi abordado anteriormente, o presente trabalho terá como finalidade responder a seguinte pergunta-problema. Qual a ação do Pilates sobre a capacidade funcional de seus usuários? Com este estudo, objetivamos verificar a capacidade funcional e o nível de atividade funcional dos usuários que praticam o método Pilates.

\section{Metodologia}

O estudo é uma pesquisa transversal de campo do tipo exploratória com abordagem quantitativa. A pesquisa transversal é feita em um breve período de tempo, em um momento determinado, ou seja, como hoje, agora. Já a pesquisa quantitativa é aquela que trabalha em forma de números e emprega técnicas rígidas e meios para analisá-los e classificá-los, tais como o seu percentual, o desvio padrão, a média entre outros (Fontelles et al 2009).

Foi realizada uma pesquisa no mês de outubro de 2020 no município de Aracati - CE com os usuários do Pilates, de ambos os sexos. Em duas clínicas que trabalham com Pilates. A clínica de Júlio Marques e Satori.

Foram selecionadas pessoas na forma aleatorizada estratificada e entregue um termo de consentimento livre e esclarecido (TCLE), foram tiradas todas as dúvidas dos participantes e a pesquisa só foi realizada mediante a assinatura do termo, sendo duas vias uma fica com o participante e outra foi entregue para o pesquisador e o participante teve um prazo de 10 dias para entregar o termo. Critérios de inclusão e exclusão: Foram incluídos no estudo praticantes do 
método Pilates, de ambos os sexos, que aceitaram participar da pesquisa e que estavam no Pilates por pelo menos 2 meses. Foram excluídos do estudo aqueles que se apresentava alguma disfunção física, cognitiva ou psicológica grave, aqueles que se ausentaram no dia da coleta de dados e que se recusaram a participar.

$\mathrm{O}$ instrumento de coleta dos dados, foi através de dois questionários sendo, ele o IPAQ e o $\mathrm{HAQ}$, por sua vez o IPAQ contém 8 perguntas que são voltadas para medir a avaliação funcional dos participantes e o tempo que se gasta realizando a atividade física, classificando-as em moderadas e vigorosas e o HAQ que contém 20 perguntas todas objetivas, com as seguintes opções: sem qualquer, com alguma, com muita ou incapaz de fazer, utilizadas para a capacidade funcional.

A população de usuários das clínicas de Pilates citada na cidade de Aracati - CE, foram utilizados todos, sendo assim selecionados 20 participantes e desse total não houve nenhuma desistência ou que se recusaram a participar da pesquisa, portanto foram avaliados quanto ao HAQ e o IPAQ, bem como algumas perguntas relacionadas ao tempo de tratamento, sexo e idade.

A pesquisa acompanhou o rito e doutrinações estabelecido pela resolução №466/2012 do Conselho Nacional de Saúde - CNS. Riscos que a pesquisa acarretou para os participantes foram mínimos, entre eles está o constrangimento que o participante poderá sofrer mediante a pesquisa, porém para minimizar esse risco foi explicado para o participante que não terá a exposição de seus dados bem como foi realizado numa sala reservada e com a porta fechada, ambiente climatizado e agradável, com cadeiras para melhor acomodação do(a) participante para responder ao questionário, para executar essa coleta de dados o participante poderá levar um acompanhante e ficará dentro da sala o participante com seu acompanhante, o pesquisador principal e o auxiliar e que os dados que foram coletados ficarão em sigilo e que poderá desistir da pesquisa se não estiver se sentindo confortável. A pesquisa foi submetida ao comitê ética da Faculdade do Vale do Jaguaribe - FVJ na data de 26/01/2020, sob o número de parecer 4. 123.633 recebeu a aprovação na data de 30/06/2020.

A análise dos dados foi realizada através do programa Microsoft Excel (2010), com o intuito central de trabalhar a explanação em tabelas e gráficos dos resultados do questionário, bem como outras informações que foram explanadas em forma de estatística descritiva.

\section{Resultados e Discussão}

Com relação a idade, sexo e tempo de tratamento, foi observado que o público feminino é muito atuante com $95 \%$ e em seguida o masculino com 5\%, com idade média 33 variando entre 14,16 anos, com o tempo de tratamento médio entre quize, conforme a tabela 1.

Tabela 1 Idade, sexo e tempo de tratamento.

\begin{tabular}{|c|c|c|c|}
\hline Idade & $33 \pm 14.16$ & & \\
\hline \multicolumn{4}{|l|}{ Sexo } \\
\hline & Feminino & $n=15$ & $95 \%$ \\
\hline & Masculino & $n=5$ & $5 \%$ \\
\hline \multicolumn{2}{|c|}{ Tempo de tratamento } & $15.5 \pm$ & \\
\hline
\end{tabular}

No questionário SHAQ, com relação a capacidade funcional foi identificado 14 pessoas, sendo $79 \%$ do sexo feminino e $60 \%$ do sexo masculino sem qualquer incapacidade, 6 pessoas apresentaram algum grau de incapacidade, correspondendo a $21 \%$ do sexo feminino e $40 \%$ do sexo masculino e por fim não foi identificado pessoas com muita incapacidade ou com incapacidade grave em ambos os sexos, conforme a tabela 2. 
Tabela 2 SHAQ questionnaire.

\begin{tabular}{llll}
\hline Sem qualquer incapacidade & $\mathrm{n}=14$ & Feminino & $79 \%$ \\
& & Masculino & $60 \%$ \\
Com alguma incapacidade & $\mathrm{n}=6$ & Feminino & $21 \%$ \\
& & Masculino & $40 \%$ \\
Com muita incapacidade & $\mathrm{n}=0$ & Feminino & $0 \%$ \\
& & Masculino & $0 \%$ \\
Incapacidade grave & $\mathrm{n}=0$ & Feminino & $0 \%$ \\
& & Masculino & $0 \%$ \\
\hline
\end{tabular}

Segundo Pacheco et al (2017) em uma revisão sistemática, onde realizou-se uma busca eletrônica nas bases LILACS, Google acadêmico, Portal Periódicos das Capes, PubMED, SciELO, que aborda pilates e flexibilidade, sendo a maior parte do estudo conduzida com pessoas jovens, em seus resultados encontrou que o método pilates mostrou-se eficaz não somente na melhora da flexibilidade, como também na resistência muscular, equilíbrio e propriocepção, obtendo resultados relevantes como o aumento da qualidade de vida, consequentemente aumento da capacidade funcional e redução do risco de lesões.

De acordo com o estudo de Valente et al (2009), que teve como objetivo em seu estudo verificar como o programa de exercícios do método pilates influencia na capacidade funcional em portadores de lombalgia crônica e obteve o seguinte resultado quanto a capacidade funcional, que os exercícios apresentam uma combinação de atividades de força e flexibilidade, visando a manutenção e desenvolvimento do equilíbrio muscular, contribuindo com a estabilização da região lombar.

Em seguida no questionário IPAQ com relação a quantos dias da última semana a pessoa caminhou por pelo menos 10 minutos contínuos, foi observado o seguinte resultado, em 2 dias na semana somente 2 pessoas realizaram caminhada, em 3 dias, foram 10 pessoas, em 4 dias, 4 pessoas, 5 dias, 1 pessoa somente e em 7 dias, 3 pessoas. Com relação aos dias em que a pessoa caminhou por pelo menos 10 minutos contínuos o tempo no total que foi gasto caminhando por dia, ou seja, a média/desvio padrão de tempo foi de 60+_ 18.3, com relação a quantos dias da última semana a pessoa realizou atividades moderadas por pelo menos 10 minutos contínuos como por exemplo, exercícios aeróbicos, bicicleta, natação ou corrida, em 1 dia na semana somente 1 pessoa realizou, 2 dias na semana, 13 pessoas realizaram, em 3 dias, 2 pessoas e em 7 dias, 4 pessoas, então a média/desvio padrão de tempo foi de 60+_20.1. Agora com relação as atividades vigorosas realizadas por pelo menos 10 minutos contínuos foram analisadas que em nenhum dia da semana 10 pessoas não realizaram a atividade, em 1 dia, 6 pessoas realizaram, em 2 dias, 3 pessoas e em 3 dias 1 pessoa, de acordo com o que foi analisado a média foi de 15+_23.4. E por fim de acordo com o tempo que foi gasto sentado durante um dia de semana a média foi de $120+23.4$ e com relação ao tempo gasto sentado durante os finais de semana a média foi de $120+$ 34.15. Conforme a tabela 3.

Segundo Mendes et al (2015), em seu estudo que teve como objetivo analisar e comparar na pré e pósintervenção a funcionalidade e a flexibilidade de grupos de mulheres adultas e idosas submetidas a exercícios de pilates no solo e obteve o seguinte resultado, que o pilates deve ser praticado para ajudar no desenvolvimento do controle do centro do organismo, com a integração da musculatura estabilizadora para criar força e equilíbrio durante atividades estáticas e dinâmicas, melhorando a funcionalidade. 
Tabela 3 IPAQ.

\begin{tabular}{lll}
\hline $1 a$ & & \\
2 dias na semana & caminhada & $\mathrm{n}=2$ \\
3 dias na semana & caminhada & $\mathrm{n}=10$ \\
4 dias na semana & caminhada & $\mathrm{n}=4$ \\
5 dias na semana & caminhada & $\mathrm{n}=1$ \\
7 dias na semana & caminhada & $\mathrm{n}=3$
\end{tabular}

$1 b$

Média/desvio padrão de tempo

$60 \pm 18.3$

$2 a$

1 dia na semana

exercícios aeróbicos: bicicleta, natação ou corrida

$\mathrm{n}=1$

2 dias na semana

exercícios aeróbicos: bicicleta, natação ou corrida

$\mathrm{n}=13$

3 dias na semana

exercícios aeróbicos: bicicleta, natação ou corrida

$n=2$

7 dias na semana

exercícios aeróbicos: bicicleta, natação ou corrida

$n=4$

$2 b$

Média/desvio padrão de tempo

$60 \pm 20.1$

$3 a$

Nenhum dia

práticas de esportes, serviços com pesos elevados

$\mathrm{n}=10$

1 dia da semana

práticas de esportes, serviços com pesos elevados

$\mathrm{n}=6$

2 dias da semana

práticas de esportes, serviços com pesos elevados

$n=3$

3 dias da semana

práticas de esportes, serviços com pesos elevados

$n=1$

$3 b$

Média/desvio padrão de tempo

$15 \pm 26.5$

4a

Média/desvio padrão de tempo

$120 \pm 23.4$

$4 b$

Média/desvio padrão de tempo

$120 \pm 34.15$

\section{Considerações Finais}

Diante dos dados coletados e do que foi abordado no presente estudo, foi possível verificar que o método pilates é bastante eficaz, contribuindo na melhora da qualidade de vida de seus praticantes assíduos, como por exemplo, 
melhorando a flexibilidade, força muscular, equilíbrio, alivia as dores, melhora a postura. Ficando notório que o público de maior ênfase são os idosos sendo mais mulheres com idades entre 60 e 65 anos, embora ainda se faça necessário mais estudos acerca do assunto para que se tenha mais comprovação da eficácia do método.

\section{Declaração de conflito de interesse}

Os autores declaram que não há conflitos de interesse.

\section{Financiamento}

Esta pesquisa não recebeu nenhum apoio financeiro.

\section{Referências}

Bueno MBT, Neves FB (2019) Efeitos do Método Pilates em Idosos: Revisão de literatura. Revista Saúde e Desenvolvimento Humano 7:99-105.

Elizabete RJ, Marcondes TFG, Liberli R, Artaxo MIN, Brandão HD, Lopes CR (2013) Perfil de qualidade de vida em praticantes de pilates. Fisioterapia Brasil 14:24-36.

Fontelles MJ, Simões MG, Farias SH, Fontelles RGS (2009) Metodologia de pesquisa ciêntifica: Diretrizes para elaboração de um protocolo de pesquisa. Fisioterapia Movimento 22:449-455.

Marés G, Batista KO, Piazza MC, Preis C, Neto LB (2012) A importância da estabilização central no método Pilates: uma revisão sistemática. Fisioterapia Movimento 25:445-451.

Mendes PMV, Santos LE, Mesquita LSA, Carvalho FT (2015) Análise da funcionalidade e flexibilidade de mulheres em exercícios de pilates no solo. Arquivo Ciência Saúde 22:53-57.

Pacheco JFR, Guimarães ACA, Kraeski MH, Kraeski AC, Souza MC, Araujo CCR (2017) Pilates e flexibilidade: Uma revisão. Revista Brasileira de Ciências da Saúde 21:275-280.

Pinto AH, Lange C, Pastore CA, Llano PMP, Castro DP, Santos F (2016) Capacidade funcional para atividades da vida diária de idosos da estratégia de Saúde da família da zona rural. Ciência \& Saúde Coletiva 21:3545-3555.

Pires DC, Sá CKC (2005) Pilates: notas sobre aspectos históricos, princípios, técnicas e aplicações. Revista Digital Buenos Aires 90:1628.

Silva ACLG, Mannrich G (2009) Pilates na reabilitação: uma revisão sistemática. Fisioterapia Movimento 22:449-455.

Valente TA, Schossler A, Bittencourt DC, Strassburger MJ (2013) Efeitos dos exercícios do método pilates em pacientes com dor lombar crônica. Revista Contexto \& Saúde 8:37-41. 